\title{
Characterizing Topological Order in Superconducting Systems
}

\author{
M. Cristina Diamantin* and Pasquale Sodand \\ Dipartimento di Fisica, University of Perugia, \\ via A. Pascoli, I-06123 Perugia, Italy \\ and \\ Sezione INFN, Perugia, Italy
}

(Dated: November 3, 2018)

\begin{abstract}
Two established frameworks account for the onset of a gap in a superconducting system: one is based on spontaneous symmetry breaking via the Anderson-Higgs-Kibble mechanism, and the other is based on the recently developed paradigm of topological order. We show that, on manifolds with non trivial topology, both mechanisms yield a degeneracy of the ground state arising only from the incompressibility induced by the presence of a gap. We compute the topological entanglement entropy of a topological superconductor and argue that its measure allows to distinguish between the two mechanisms of generating a superconducting gap.
\end{abstract}

PACS numbers: 11.10.z;74.20.Mn;05.30.Pr

\section{INTRODUCTION}

The discovery of the fractional quantum Hall liquids (FQHLs) [1] led to a new understanding of strongly correlated electron systems and to the idea of a new type of quantum order [2], called topological order. Quantum order in general describes the zero-temperature properties of strongly entangled quantum ground states not arising from spontaneous symmetry breaking (SSB). A very surprising property exhibited by FQHLs is that, due to repulsive interactions and strong correlations between electrons, they give rise to an incompressible state even when the first Landau level is only partially filled at some "magical" filling fraction and all bulk excitations have a finite gap; no order parameter is available for this state. To explain these features Wen [2] introduced the paradigm of topological order as a special type of quantum order whose hallmarks are the presence of a gap for all excitations (incompressibility) and the degeneracy of the ground state on manifolds with non-trivial topology. Due to incompressibility, at low energies, the dynamical degrees of freedom of a FQHLs are only gapless edge excitations, which may be used to characterize the various topological orders [2]. For fractional quantum Hall systems another important hallmark is the presence of excitations carrying fractional charge and fractional spin and statistics [3].

The long distance behavior of systems exhibiting topological order is usually described by incompressible fundamental (e.g. single-layer FQHLs) or non-fundamental (e.g. double-layer FQHLs) fluids. The simplest example of a $2 D$ topological fluid [2] is characterized by a ground-state described by a low-energy effective action given solely by the topological Chern-Simons (CS) the-

\footnotetext{
*Electronic address: cristina.diamantini@pg.infn.it
}

${ }^{\dagger}$ Electronic address: pasquale.sodano@pg.infn.it ory [4]:

$$
S=k / 4 \pi \int d^{3} x A_{\mu} \epsilon^{\mu \nu \alpha} \partial_{\nu} A_{\alpha}
$$

describing a compact $U(1)$ gauge field $A_{\mu}$ whose dual field strength $F^{\mu}=\epsilon^{\mu \nu \alpha} \partial_{\nu} A_{\alpha}$ is interpreted as the conserved matter current; the degeneracy of the ground state on a manifold of genus $g$ is given by $k^{g}$ (or $\left(k_{1} k_{2}\right)^{g}$ if $k=k_{1} / k_{2}$ is a rational number).

In 5] a superconductivity mechanism based on a topologically ordered ground state has been proposed. The dominant term in the action describing these superconductors is the topological BF action [6]. The BF action, in $(\mathrm{D}+1)$ dimensions, describes the coupling between an antisymmetric tensor field $B_{p}$ and the curvature $F=d A_{D-p}$ of a tensor $A_{D-p}$, and it is given by:

$$
S=k / 2 \pi \int d^{(D+1)} B_{p} \wedge d A_{D-p} .
$$

In its application to superconductivity the action given in eq.(2) is invariant under P-and T- symmetry and is related to non-chiral fundamental incompressible fluids. A remarkable realization of this mechanism of superconductivity is provided by Josephson junction arrays (JJAs) [7]. In fact, the action of JJAs can be exactly mapped onto an Abelian gauge theory with two gauge fields describing a current of charges and a current of vortices coupled by a mixed Chern-Simons term [8]:

$$
\mathcal{L}=-\frac{1}{4 e^{2}} F_{\mu \nu} F^{\mu \nu}+\frac{\kappa}{2 \pi} A_{\mu} \epsilon^{\mu \alpha \nu} \partial_{\alpha} B_{\nu}-\frac{1}{4 g^{2}} f_{\mu \nu} f^{\mu \nu} .
$$

We refer to this model as a fundamental BF model, since (3) is derived from the microscopic model and does not describe only the low energy degrees of freedom as is the case of effective theories. The Abelian gauge theory exactly reproduces the phase diagram of JJAs and the insulator/superconductor quantum phase transition at $\mathrm{T}=0$ 7]. The insulating phase turns out to be dual to the superconducting phase and it is also topological. Global 
superconductivity in planar JJA is thus the simplest example of the mechanism of superconductivity proposed in [5]. JJA have also been recently considered by several other authors [9], as controllable devices exhibiting topological order. Of course, planar JJAs with open boundary conditions do not exhibit ground state degeneracy. To see a ground state degeneracy one should require periodic boundary conditions implementing a torus topology; toroidal arrays are not difficult to realize in practice [10].

Abelian effective CS theories are only one of the two candidates to describe the long-distance properties of fundamental topological fluids: the other is given by $W_{1+\infty}$ minimal models; indeed, Abelian CS theories describe special cases of quantum incompressible fluids, but not all of them. As evidenced in [11, the $W_{1+\infty}$ minimal models are not described by Abelian CS theories since, while all the Abelian CS theories have a dynamical $W_{1+\infty}$ symmetry algebra, the contrary is not true.

It should be stressed that $W_{1+\infty}$ models imply nonAbelian fractional statistics even for fundamental quantum Hall fluids, although they are not described by nonAbelian CS models. Intuitively, a model of a topological fluid based on the $W_{1+\infty}$ algebra is "more incompressible" than its CS counterpart since it supports less degrees of freedom and yields a different entanglement entropy. Non-Abelian CS models have been proposed, indeed, as effective theories for composite, nonfundamental quantum incompressible fluids, e.g. doublelayer quantum Hall systems; as such they are not best suited to study the fundamental mechanism at the origin of quantum incompressibility. As evidenced in [12], non-Abelian CS models can be viewed as cosets of $W_{1+\infty}$ fundamental models and this naturally reflects their composite character.

Topological field theories emerge naturally also in describing superconductors. It is well known 13] that the degrees of freedom of an Abelian $U(1)$ Higgs model spontaneously broken to $Z_{N}$ ( $N=2$ for conventional superconductivity) are charges ne $(n=0 \ldots \ldots . N-1)$ and vortices $\frac{k 2 \pi}{N e}(k=0 \ldots . . N-1)$. While Coulomb interactions are screened in the broken phase, Aharonov-Bohm interactions are not and the latter are described by effective $\mathrm{BF}$ theories described by the action given in eq.(2). In $(2+1)$ dimensions eq. (2) is the action of a mixed CS model eq.(11); here, the gap and the ground state degeneracy are due to the breaking $U(1) \rightarrow Z_{N}$.

Mixed CS models have recently attracted a wide interest 14] to describe topological fluids in various contexts. As already mentioned, Abelian mixed ChernSimons models emerge naturally as an effective theory of the low-energy long -distance properties of superconductors no matter what is the microscopic mechanism by which the gap is opened. In this paper we shall evidence that, for superconducting systems, quantum incompressibility alone (i.e. the emergence of a gap) is enough to account for all the features normally associated with topological order in $2 D$, like the emergence of a ground state degeneracy on spaces with non trivial topology and of excitations with fractional charge and statistics. The gap can be of different origin: SSB or topological, but we will show that this has no influence on the kinematics of the resulting incompressible fluid.

There is a difference in the ground state degeneracy between topological and standard superconductors 15] and this allows to distinguish between the two microscopic mechanisms by which the superconducting gap is opened: indeed, for topological superconductors the ground state degeneracy is entirely determined by the coefficient $k$ of the BF action while, for standard superconductors, the ground state degeneracy is due entirely to the SSB of $U(1) \rightarrow Z_{N}$. As a result, there is a remarkable difference in the ground state degeneracy exhibited by the standard superconductors in $(2+1)$ dimensions analyzed in [16] and the topological superconductors analyzed in [5]: this is indeed relevant since, as we show in this paper, by computing the entanglement entropy of the ground state [17], it becomes possible to distinguish between topological and standard superconductors. Since an experimental footprint of the entanglement entropy may be observed in transport measurements in strongly correlated systems [18], one may envisage that similar effects may be evidenced in toroidal JJA's.

Our subsequent analysis is based on the observation that, once there is a gap, no matter what is its origin, the ground state has the universal properties of a fundamental quantum incompressible fluid characterized by a dynamical $W_{1+\infty}$ symmetry algebra (or $W_{1+\infty} \otimes \bar{W}_{1+\infty}$ if they are not chiral as in the BF model we are interested in) for the edges excitations both for topological and standard superconductors. As already pointed out in [19], the symmetry under quantum area preserving diffeomorphisms arising from the tquantum incompressibility alone is enough to account for the emergence of both a ground state degeneracy and fractional spin and statistics. Thus, looking only at the edges excitations does not provide useful information to distinguish between topological and standard superconductors; at variance, counting the ground state degeneracy provides a way to characterize these two very different microscopic mechanism of opening a superconducting gap.

In section II we analyze incompressible fluids on a torus: our aim here is to show that ground-state degeneracy arises only from incompressibility. For this purpose we show that, no matter how the gap is opened, once one has an incompressible fluid on a torus, the emerging dynamical algebra, namely the Fairlie, Fletcher and Zachos trigonometric algebra [20], is -by itself- enough to account for the ground state degeneracy; as a result, every incompressible fluid has ground state degeneracy on a torus. We evidence how the $W_{1+\infty}$ algebra emerges as a dynamical quantum symmetry group of Abelian CS theories and how the $W_{1+\infty} \otimes \bar{W}_{1+\infty}$ arises for the BF model. We then argue that, for pure CS theories, it is possible to relate directly the generators of the large gauge transformations (determining the ground state degeneracy of 
a CS theory [21]) with those of the area preserving diffeomorphisms. Finally, we show that the ground state degeneracy is affected by the how the gap originates. In Section III we analyze incompressible fluids on manifolds with boundaries: there, we show that the Abelian mixed CS theory possess the full $W_{1+\infty}$ dynamical symmetry algebra. Moreover, using the holographic partition functions, we show that,despite the fact that once the gap is opened both superconductors share the properties of an incompressible fluid, a measure of the topological entanglement entropy may distinguish between the two. Section $I V$ is devoted to a few concluding remarks.

\section{INCOMPRESSIBLE FLUIDS ON A TORUS}

The configurations of $2 D$ incompressible fluids are spanned by area-preserving diffeomorphisms. As a result, the dynamical symmetry algebra of quantum incompressible fluids is given by $W_{1+\infty}$, which is the quantization of area-preserving diffeomorphisms. In this section we show that, for a torus topology and for both chiral and non-chiral fluids, the dynamical algebra alone is responsible for the ground-state degeneracy, independently of the origin of the gap and that, using the algebra $W_{1+\infty}$ [11], allows to determine the bulk and edge excitations of a quantum incompressible fluid.

We start by noticing that incompressibility implies that one may regard the original coordinate space as the phase space, i.e. $\left(p, q \equiv x_{1}, x_{2}\right)$ since, from Liouville' $\mathrm{s}$ theorem, one knows that canonical transformations should preserve the volume in phase space. On this space, canonical transformations are defined as $\delta q=$ $\{q, F(p, q)\}=\partial F / \partial p, \delta p=\{q, F(p, q)\}=-\partial F / \partial q$, where $\left(x_{1}, x_{2}\right)$ are the space coordinates and $\{$,$\} is the$ Poisson bracket. On a plane, a basis of generating functions may be given in terms of the complex coordinates $z=x_{1}+i x_{2}, \bar{z}=x_{1}-i x_{2}$, as

$$
F_{n, m}^{c l}=z^{n} \bar{z}^{m}
$$

which satisfy the classical $w_{\infty}$ algebra of area-preserving diffeomorphisms. On a square torus ( i.e. a torus with equal sides $2 \pi$ ), instead, the generators are given by a set of complete harmonics

$$
F_{n, m}^{c l}=\exp i\left\{x_{1} n+x_{2} m\right\},
$$

with $n, m$ integers. Thus, at the classical level, one gets the algebra $\left\{F_{\bar{n}}^{c l}, F_{\bar{m}}^{c l}\right\}=\bar{n} \times \bar{m} F_{\bar{n}+\bar{m}}^{c l}$.

To quantize this algebra one may consider a generic torus of dimensions $L_{x}, L_{y}$ and ( with a pertinent normalization) assume the following commutation relation

$$
\left[\frac{x}{L_{x}}, \frac{y}{L_{y}}\right]=\frac{i}{2 \pi k},
$$

where $k$ is a dimensionless constant giving the ratio between the volume of the torus and the volume of the unit cell in phase space. In the following we assume $k=k_{1} / k_{2}$, with $k_{1}$ and $k_{2}$ integers. If one regards $\frac{x}{L_{x}}$ as a coordinate and $\frac{y}{2 \pi k L_{y}}$ as a momentum in the phase space, the generators may be written as

$$
K_{m, n}=\exp 2 \pi i\left\{\frac{x}{L_{x}} n+\frac{y}{L_{y}} m\right\}
$$

with commutation relations given by

$$
\left[K_{m, n}, K_{p, q}\right]=2 i \sin \left[\frac{2 \pi}{k}(m q-n p)\right] K_{m+p, n+q},
$$

which is the $W_{1+\infty}$ algebra on the torus, also called the Fairlie Fletcher Zachos trigonometric algebra [20]. States of the quantum incompressible fluid must then fall into representations of this algebra.

To study the representations of the $W_{1+\infty}$ algebra one observes that

$$
K_{m, n} K_{p, q}=\exp \left[\frac{2 \pi i}{k}(m q-n p)\right] K_{p, q} K_{m, n}
$$

thus, the algebra admits a non-trivial two-cocycle and, if $k=k_{1} / k_{2}$, the generator $K_{k_{1} m, k_{1} n}$ is a Casimir operator for the algebra. When $k$ is rational, if one applies the generator $K_{m, n}$ to a physical state $\psi(x)$, one gets

$$
K_{m, n} \psi(x)=\exp \left[\frac{i m x}{k L_{x}}\right] \exp \left[\frac{m n \pi i}{k}\right] \psi(x+2 \pi n),
$$

so that

$$
\begin{aligned}
K_{k_{1} m, k_{1} n} \psi(x) & =\exp \left[\frac{i m k_{2} x}{L_{x}}\right] \times \\
& \times \exp \left[i m n k_{1} k_{2} \pi\right] \psi\left(x+2 \pi k_{1} n\right) \\
& =\exp i \phi_{k_{1} k_{2}} \psi(x),
\end{aligned}
$$

where $\phi_{k_{1} k_{2}}=2 \pi k_{1} k_{2}\left(m \theta_{1}+n \theta_{2}\right)$. In fact, the action of a Casimir operator on the physical state can change it only by a phase, which is fully specified by the two angles $\theta_{1}$ and $\theta_{2}$. As a result, one may write the wave function as

$$
\psi(x)=\sum_{p} \exp \left[\frac{i p x}{L_{x}}\right] \exp \left[\frac{i \theta_{1} k_{2} x}{L_{x}}\right] c(p)
$$

where $c(p)$ satisfies the quasi-periodicity condition $c(p-$ $\left.m k_{2}\right)=\exp \left[i \theta_{2} m k_{1} k_{2}\right] c(p)$, as it can be seen by applying the Casimir to $\psi(x)$. Thus, for each $k_{1}$, one has $k_{2}$ independent states implying a degeneracy given by $k_{1} k_{2}$ on the torus while, on a surface of genus $g$, it is given by $\left(k_{1} k_{2}\right)^{g}$. We have thus shown that incompressibility alone leads to degeneracy of the ground state We point out that this degeneracy is only a kinematic consequence of quantum incompressibility and has nothing to do with the dynamical origin of the gap from which incompressibility arises.

We will now show how $W_{1+\infty}$ and $W_{1+\infty} \otimes \bar{W}_{1+\infty}$ algebras arise generically as the dynamical symmetry group 
of Abelian CS theories and BF theories respectively. For the former we focus on the global time-independent symmetries of the CS action since they are the symmetries of the configuration space. Quantum states fall then into representations of this symmetry group, the action being represented by time-independent charges.

The largest time-independent symmetry group of a CS gauge theory is given by global time-independent gauge transformations and spatial diffeomorphisms. Since the CS action is topological, for compact surfaces, only gauge transformations survive. This can be easily seen if one recalls that gauge fields behave as a phase space and satisfy commutation relations given by $\left[A_{i}(x), A_{j}(y)\right]=$ $(i 2 \pi / k) \epsilon_{i j} \delta(x-y)$, where $i=1,2$ label the space coordinate of the manifold. For CS models defined on manifolds with boundaries the relationship with a $W_{1+\infty}$ algebra was first established for a torus in [22], using the equivalence between a CS model and the Landau problem. Here, we follow a different approach and show that, for pure CS model, it is possible to relate directly the generators of the large gauge transformations with those of the area preserving diffeomorphisms.

We start by decomposing the spatial component of the gauge field into global and local degrees of freedom. In fact, the contributions of these two parts decouple in the action. Following [21, 23] we decompose the gauge field $A$ into exact, co-exact and harmonic parts as

$$
A=d \phi+\delta \chi+h ; h=2 \pi \sum_{i=1}^{g}\left(u_{i} \alpha_{i}+v_{i} \beta_{i}\right)
$$

We restrict ourselves to $g=1$ with $\alpha$ and $\beta$ canonical harmonic 1-forms of the surface (dual to a canonical homology basis). The commutation relations between the gauge fields imply that

$$
\begin{aligned}
{[\delta \chi(x), \phi(y)] } & =\frac{2 \pi}{i k} \delta^{2}(x-y) \\
{\left.\left[v_{i}, u_{j}\right)\right] } & =\frac{1}{2 \pi i k} \delta_{i j}
\end{aligned}
$$

with $k$ the CS coefficient. Eq.(14) tells then that $\chi$ and $v$ may be regarded as momenta. Finally, requiring that the Gauss law annihilates physical states, one gets a quantum theory which is gauge invariant under small gauge transformation. Thus, the configuration space is reduced to a $g$-dimensional (1-dimensional if, as in our derivation, $g=1)$ space with coordinates $u_{i}(i=1$ when $g=1)$.

Large gauge transformations $u \rightarrow u+n ; v \rightarrow v+m$ are generated by

$$
U_{m, n}=\exp \left\{\frac{\partial}{\partial u} n+2 i \pi k m\right\}
$$

and satisfy the algebra

$$
\left[U_{m, n}, U_{p, q}\right]=2 i \sin \{\pi k(n p-m q)\} U_{m+p, n+q},
$$

which is nothing else than (8) with the identification: $\frac{x}{L_{x}}=2 \pi k u$ and $\frac{y}{L_{y}}=-\frac{i}{2 \pi k} \frac{\partial}{\partial u}$. The generators of large gauge transformations can be identified then with the generators of $W_{1+\infty}$. The CS coefficient $k$ measures the ratio between the total flux piercing the torus and the unit flux; when there is commensurability, namely when $k=k_{1} / k_{2}$, the degeneracy of the ground state is given by $k_{1} / k_{2}$.

Let us now concentrate on the BF model and show how a $W_{1+\infty} \otimes \bar{W}_{1+\infty}$ algebra arises as the dynamical symmetry group of Abelian mixed CS theories describing topological superconductors and described by the action:

$$
S_{B F}=\frac{k}{2 \pi} \int_{M_{2+1}} A_{1} \wedge d B_{1}
$$

On the torus and let us decompose the forms $A$ and $B$ in the exact, co-exact and harmonic part:

$$
A=d \phi_{A}-* d \chi_{A}+u \alpha+v \beta, B=d \phi_{B}-* d \chi_{B}+w \alpha+r \beta,
$$

where $\alpha$ and $\beta$ form a basis of harmonic one-forms, dual to a canonical homology basis. The action eq. (17) become then

$$
2 \pi k \int_{R} r \dot{u}+v \dot{w}
$$

with commutation relations

$$
[r, u]=[v, w]=\frac{1}{2 i \pi k} .
$$

The generators of large gauge transformations can thus be written as:

$$
U(n, m, t, l)=\exp \left[n \frac{\partial}{\partial w}+m \frac{\partial}{\partial u}-2 \pi k(t w+l u)\right],
$$

and they satisfies the algebra:

$$
\begin{aligned}
& U\left(n_{1}, m_{1}, t_{1}, l_{1}\right) U\left(n_{2}, m_{2}, t_{2}, l_{2}\right)= \\
& =U\left(n_{1}+n_{2}, m_{1}+m_{2}, t_{1}+t_{2}, l_{1}+l_{2}\right) \times \\
& \times \exp \left[i \pi k\left(t_{1} n_{2}+l_{1} m_{2}-n_{1} l_{2}-m_{1} t_{2}\right)\right] .
\end{aligned}
$$

We note that for $k$ an even integer the cocycle is always trivial.

This case corresponds to having two incompressible fluids with coordinates $x_{i}$ and $y_{i}(i=A, B)$ corresponding to the two gauge fields $A$ and $B$ and commutation relations

$$
\left[\frac{x_{A(B)}}{L_{x}}, \frac{y_{B(A)}}{L_{y}}\right]=\frac{i}{2 \pi k}
$$

with generators

$$
K_{\bar{m}, \bar{n}}=\exp 2 \pi i\left\{\frac{x_{i}}{L_{x}} n_{i}+\frac{y_{i}}{L_{y}} m_{i}\right\} .
$$

With the identification: $\frac{x_{A}}{L_{x}}=2 \pi k u, \frac{y_{A}}{L_{y}}=-i \frac{\partial}{\partial u}$ and $\frac{x_{B}}{L_{x}}=2 \pi k w$ and $\frac{y_{B}}{L_{y}}=-i \frac{\partial}{\partial w}$, the generators of large 
gauge transformations can be identified then with the generators of $W_{1+\infty} \otimes \bar{W}_{1+\infty}$ algebra.

The generators (23) can be always be rewritten as:

$$
K_{\bar{m}, \bar{n}}=K_{n_{+}, m_{+}} K_{n_{-}, m_{-}},
$$

with

$$
\begin{aligned}
& K_{n_{+}, m_{+}}=\exp 2 \pi i\left\{n_{+}\left(\frac{x_{A}}{L_{x}}+\frac{x_{B}}{L_{x}}\right)+m_{+}\left(\frac{y_{A}}{L_{y}}+\frac{y_{B}}{L_{y}}\right)\right\} \\
& K_{n_{-}, m_{-}}=\exp 2 \pi i\left\{n_{-}\left(\frac{x_{A}}{L_{x}}-\frac{x_{B}}{L_{x}}\right)+m_{-}\left(\frac{y_{A}}{L_{y}}-\frac{y_{B}}{L_{y}}\right)(2\right.
\end{aligned}
$$

¿From eq.(24) we clearly see that $K_{n_{+}, m_{+}}$and $K_{n_{-}, m_{-}}$ are two sets of commuting operators corresponding to the combination $\frac{x(y)_{A}}{L_{x(y)}}+\frac{x(y)_{B}}{L_{x(y)}}$ and $\frac{x(y)_{A}}{L_{x(y)}}-\frac{x(y)_{B}}{L_{x(y)}}$. In this case we thus have a $W_{1+\infty} \otimes \bar{W}_{1+\infty}$ algebra thereby giving a degeneracy on a torus $\left(k_{1} k_{2}\right) \times\left(k_{1} k_{2}\right)$. This corresponds exactly to the degeneracy we expected for a mixed CS theory when both gauge fields are compact. In [15] we have however shown that the superconducting phase of the mixed CS theory has a ground state degeneracy that is only $\left(k_{1} k_{2}\right)$ because due to the confinement of magnetic vortices one of the two gauge fields behaves as noncompact. ¿From the point of view of the $W_{1+\infty} \otimes \bar{W}_{1+\infty}$ algebra, this corresponds to the fact that only one of the two chirality survives.

Superconducting systems allow to realize that, even if a ground state degeneracy should be always present due to the emergence of a gap (incompressibility), there is still a remarkable difference between models in which the gap arises as a result of SSB and models where it originates from topology; in fact, for the latter situation, the ground state degeneracy is characteristic of the theory independently of which phase is realized while, in the former situation, the ground state degeneracy appears only after SSB.

There is also another fundamental difference between these two superconductors. When the gap arises from SSB, the degeneracy is determined by the breaking $U(1) \rightarrow Z_{N}$ : in [16] the $U(1)$ symmetry is spontaneously broken down to $Z_{2}$ by Cooper pair formation and the ground state degeneracy is 4 ; the residual AharonovBohm interactions between charges and vortices can be described here by an effective mixed CS model with $k=2$ [16]. The degeneracy $k^{2}$ corresponds, in the topological $\mathrm{BF}$ model [15], to the case in which both topological defects, electric and magnetic, are in a dense phase. However this phase is not allowed dynamically and topological superconductors corresponds to a phase in which only an electric condensate is present and the ground state degeneracy is $k$ or $k_{1} k_{2}$ if $k=k_{1} / k_{2}$. For instance, since planar JJAs are described by a fundamental CS theory with two coupled gauge fields [7], one finds that the degeneracy on a torus is one for the unfrustrated model, which is described by a mixed CS model with CS coefficient $k=1$, and two for the fully frustrated model, which has $k=1 / 2$ [5].
Since the degeneracy on the torus is equivalent to the number of particle types [8] and the quantum dimension of each type of particle is one for Abelian systems, this difference in the ground state degeneracy between the two superconductors leads to a different topological entanglement entropy [17] in the ground state, as we will show with a more formal argument in the next section. In fact topological entanglement entropy is defined as [17] $-\gamma=\log \mathcal{D}$ where $\mathcal{D}=\sqrt{\sum_{a} d_{a}^{2}}$ is the total quantum dimension and $d_{a}$ is the quantum dimension of a particle of charge $a$, and for a system with $k$ or $k^{2}$ type of particles each of quantum dimension $d=1$, we will have $\gamma=\log \sqrt{k}$ and $\gamma=\log k$ respectively. For topological superconductors the entanglement entropy is thus half of the one of superconductors originating from SSB. A measurement of the topological entanglement entropy can thus distinguish between the two.

\section{MANIFOLDS WITH BOUDARIES}

On a disk $D$, the classical generators of area preserving diffeomorphism algebra, $w_{\infty}$, are given by eq.(4). At the quantum level the generators of $w_{\infty}, W_{1+\infty}, V_{n}$, are characterized [27] by a mode index $n \in Z$ and a conformal spin $h=i+1$, and satisfy the algebra:

$$
\begin{aligned}
& {\left[V_{n}^{i}, V_{m}^{j}\right]=(j n-i m) V_{n+m}^{i+j-1}+q(i, j, m, n) V_{n+m}^{i+j-3}+} \\
& \ldots+c \delta^{i j} \delta_{m+n, 0} d(i, n),
\end{aligned}
$$

where $q$ and $d$ are pertinent [27] polynomials and $c$ is the central charge. The dots stand for a series of terms involving the operators $V_{n+m}^{i+j-1-2 k}$ [27].

The generators $V_{n}^{0}$ are associated to the charge of the edge excitations while $V_{n}^{1}$ to the angular-momentum modes; they satisfy the Kac-Moody algebra:

$$
\begin{aligned}
& {\left[V_{n}^{0}, V_{m}^{0}\right]=n c \delta_{m+n, 0}} \\
& {\left[V_{n}^{1}, V_{m}^{0}\right]=-m V_{n+m}^{0}} \\
& {\left[V_{n}^{1}, V_{m}^{1}\right]=(n-m) V_{n+m}^{1}+\frac{1}{12} c n\left(n^{2}-1\right) \delta_{m+n, 0}}
\end{aligned}
$$

All $W_{1+\infty}$ unitary, irreducible, highest-weight representations have been found by Kac [27] and applied to incompressible quantum Hall fluids by Cappelli, Trugenberger and Zemba 11]. These representations exist only for positive integer central charge $c=m=1,2, \ldots$ and, if $c=1$, they are equivalent to those of the Abelian sub-algebra $\hat{U}(1)$ of $W_{1+\infty}$, corresponding to the edge excitations of a single Abelian CS theory. If, instead, $c=2,3, \ldots$ there are two kinds of representations, generic and degenerate, depending on the weight. The generic representations are equivalent to the corresponding representations of the multi-component Abelian algebra $\hat{U}(1) .{ }^{\otimes m}$ having the same weight and correspond to the edge excitations of a multiple Abelian CS theory. The degenerate representations instead are contained in the corresponding $\hat{U}(1)^{\otimes m}$ representations, i.e. the latter being reducible $W_{1+\infty}$ representations. 
Let us consider now a CS theory defined on $D \times R$ (where $R$ accounts for time). It has been shown by Witten [28] that this theory may be quantized upon eliminating only the degrees of freedom associated to the interior of $D$. As a result, gauge transformations- which, as already noticed, may be regarded as diffeomnorphisms [29] in a CS model- relate equivalent fields only in the interior of the disc, while on $\partial D$ they play a role somewhat similar to global gauge transformations. The residual states localized on the circular boundary are the CS edge states and are equivalent to the edge excitations of a droplet of incompressible fluid. Furthermore, the generators of the gauge transformation which do not vanish on $\partial D$ generate a $U(1)$ Kac-Moody algebra isomorphic to the algebra of a chiral boson moving on $\partial D$ so that, after quantization, the edge states satisfy a Virasoro algebra with central charge $c=1$. It has been shown [1] that the generators of this Virasoro algebra commute with the boundary Hamiltonian and correspond to local coordinates transformations. In the following we show that the edge excitations generate not only the Virasoro algebra, but the full algebra $W_{1+\infty}$ : namely, there are more operators which commute with the boundary Hamiltonian but do not generate local coordinates tranformations [11]. This new result generalize to manifold with boundaries the results obtained for the torus, where the generators of large gauge transformations are in $1 \leftrightarrow 1$ correspondence with the one of the FFZ algebra.

To prove our result let us review some well known results for edges excitations [2]. Following [29], if one considers gauge transformations satisfying $\left.\Lambda\right|_{\partial D}=$ $\exp (i N \theta) N \in Z$, the commutators between global charges can be written as

$$
\left[Q_{N}, Q_{M}\right]=k N \delta_{N+M, 0} .
$$

This provides a CS construction of the Fourier modes of a massless chiral boson on a circle. The Virasoro generators may be written, using the Sugawara construction, as

$$
L_{N}=\frac{1}{2 k} \sum_{L=-\infty}^{\infty}: Q_{N-L} Q_{L}:
$$

and satisfy the algebra

$$
\left[L_{N}, L_{M}\right]=(N-M) L_{N+M}+\frac{c}{12}\left(n^{3}-n\right) \delta_{N+M, 0},
$$

with central charge $c=1$. As a result, the edge excitations of a CS gauge theory are described by the chiral boson theory [2].

To obtain the action for the chiral boson one may start from the CS action [2]:

$$
S_{C S}=\frac{k}{4 \pi} \int A_{\mu} \epsilon_{\mu \nu \alpha} \partial_{\alpha} A_{\nu}
$$

which, in the gauge $A_{0}=0 \Longrightarrow A_{i}=\partial_{i} \phi$, yields

$$
S_{E E}=\frac{k}{4 \pi} \int \partial_{t} \phi \partial_{x} \phi d x d t
$$

where $t$ is the time coordinate and $x$ is the coordinate parallel to the boundary, which is conveniently parametrized as $x=R \theta$ with $R$ being the radius of $D$. The equation describes a chiral boson with zero velocity. This is not surprising since a finite velocity of the edge excitations is a boundary effect, which may be induced by a large gauge transformation. In fact, upon fixing the gauge as $A_{0^{\prime}}=A_{0}+v A_{x}$ one gets the action

$$
S_{E E}=\frac{k}{4 \pi} \int\left(\partial_{t}+v \partial_{x}\right) \phi \partial_{x} \phi d x d t .
$$

Here $v$ is the velocity of the chiral boson. The Hamiltonian derived from eq. (32) is given by

$$
H=-\frac{k v}{4 \pi} \int_{0}^{2 \pi R} \partial_{x} \phi \partial_{x} \phi d x ;
$$

the chiral current is given by

$$
J=-1 /(2 \pi) \partial_{x} \phi=1 /(2 \pi) \sum_{n=-\infty}^{\infty} \alpha_{n} \exp (i n(\theta-v t))
$$

and the generators of the Virasoro algebra may be written as

$$
\begin{aligned}
{\left[\alpha_{n}, \alpha_{m}\right] } & =n \delta_{n+m, 0} \\
L_{n} & =\frac{k}{2} \sum_{l=-\infty}^{\infty}: \alpha_{n-l} \alpha_{l}: ; \\
{\left[L_{n}, L_{m}\right] } & =(n-m) L_{n+m}+\frac{c}{12}\left(n^{3}-n\right) \delta_{n+m, 0}
\end{aligned}
$$

with central charge $c=1$.

To get the full $W_{1+\infty}$ algebra one may use the equivalence between chiral boson and Weyl fermion in $1+1$ dimensions [30]. The chiral boson is, in fact, equivalent [30] to a Weyl fermion $\psi$ described by the Hamiltonian:

$$
H=\frac{k v}{8 \pi} \int_{0}^{2 \pi R} \psi^{\dagger}\left(i \partial_{x}\right) \psi d x+\text { h.c. }
$$

with $\psi(\theta)=1 / \sqrt{R} \sum_{l=-\infty}^{\infty} e^{i(l-1 / 2) \theta} b_{l} . \quad b_{l}$ and $b_{l}^{\dagger}$ are fermionic creation and annihilation operator satisfying $\left\{b_{l}, b_{m}\right\}=\delta_{l, m}$. In [11] it was shown that it is possible to construct the generators of the $W_{1+\infty}$ algebra in terms of these fermionic fields:

$$
\begin{aligned}
V_{n} & =\int_{0}^{2 \pi} d \theta \psi^{\dagger}(\theta): e^{-i n \theta}\left(i \partial_{\theta}\right): \psi(\theta) \\
& =\sum_{l=-\infty}^{\infty} p(l, n, i) b_{l-n}^{\dagger} b_{n}, i \geq 0
\end{aligned}
$$

where the normal ordering is done in such a way that $V_{n}^{i \dagger}=V_{-n}$ and $p(l, n, i)$ are the ith order polynomials in $l$. These generators satisfy the algebra (26) with $c=1$ and the generators: $V_{n}^{0}, V_{0}^{1}$ form the Virasoro sub-algebra. The new and, at this point rather simple, remark is that 
the Hamiltonian (35) commutes with all the generators (36) and not only with $V_{n}^{0}$ and $V_{0}^{1}$ :

$$
\left[V_{n}, H\right]=0 .
$$

The CS coefficient $k$ enters the algebra by normalizing the operators $V_{n}^{0}$ and measures the physical charge of the edge excitations since they satisfy the Kac-Moody algebra

$$
\left[V_{n}^{0}, V_{m}^{0}\right]=k n \delta_{n+m, 0} .
$$

For quantum Hall fluids the CS coefficient of the effective theory is given by $k=1 / \nu$, where $\nu$ is the filling fraction [1] and $k=1$ corresponds to the filled Landau level. Furthermore, determining the spectrum of the operators $-V_{0}^{0}$ and $V_{0}^{1}$ allows to establish that the CS coefficient $k$ contributes also to the charge $\mathrm{Q}$ and the spin $\mathrm{J}$ of the bulk excitations as $Q=q / k$ and $J=q^{2} /(2 k)$.

We want now to analyze the case of the mixed CS theory (BF) relevant for topological superconductivity, where $\mathrm{P}$-and-T symmetry are preserved, on a manifold with boundaries. Our aim is to compute, via the holographic partition function 24], the topological entanglement entropy since this quantity can distinguish between model described by a fundamental $B F$ theory from model that are described by an effective $B F$ theory like the one described in [16] after SSB. States of a ChernSimons theory are accounted for by the conformal blocks of a conformal eld theory (CFT). Primary fields of the CFT at the edge are in one-to-one correspondences with the quasiparticles in the topological phase, and ChernSimons states may be identied with the characters of the CFT. Trough the Verlinde formula [25] one can write the total quantum dimension $\mathcal{D}$ as

$$
\mathcal{D}=\frac{1}{S_{0}^{0}}
$$

where $S_{0}^{0}$ is is an element of the modular $S$ matrix corresponding to the largest eigenvalue [24].

This matrix has been computed in [26] for the mixed CS theory and we have

$$
S_{\beta}^{\beta^{\prime}}=\frac{1}{\sqrt{\left|\Lambda / \Lambda^{*}\right|}} \exp -2 \pi i\left(\beta, \beta^{c}\right),
$$

and modular partition function

$$
Z=\sum_{\beta \in \Lambda / \Lambda^{*}} \chi_{\beta} \chi_{\bar{\beta}}
$$

Here $\chi_{\beta}$ are the characters and are given by the $\left(k_{1} k_{2}\right)^{2}$ theta functions that solve the quasi-periodic condition that determine the ground state wave functions of the BF model with symmetry $U(1) \times U(1)$ with both topological defects in a dense phase. The lattice $\Lambda / \Lambda^{*}$ is a lattice whose points correspond to the sectors of the rational CFT at the edges (for $k$ rational). $\left(\beta, \beta^{k}\right)$ is a quadratic form [26] such that $\exp -2 \pi i\left(0,0^{\circ}\right)=1$. ¿From this consideration we see that although formally equivalent, namely two chiral bosons with opposite chirality, the edge theory of topological superconductors and superconductors arising from SSB leads two different topological entanglement entropy. In fact for the former we have, in the superconducting phase only $\left(k_{1} k_{2}\right)$ independent theta functions with a topological entanglement entropy $\gamma=\log k$, while the latter has $\left(k_{1} k_{2}\right)^{2}$ independent theta functions and thus a topological entanglement entropy $\gamma=2 \log k$.

The algebra of the edge excitations for PT invariant incompressible fluids with a gap arising from SSB was analyzed in [19]. Due to the PT-invariance, the algebra is still the direct product of two $W_{1+\infty}$ algebras of opposite chirality, but one has to take into account that, as a result of the fact that the $U(1)$ gauge symmetry is spontaneously broken, the charge ceases to be a good quantum number in the ground state of the broken phase. Since the quantum $W_{1+\infty}$ algebra contains a $\hat{U}(1)$ KacMoody current $V_{n}^{0}$, one can identify the electric charge current with the diagonal vector current $V_{n}^{0}+\bar{V}_{n}^{0}$. This Kac-Moody symmetry has to be divided by the dynamical symmetry group, yielding the a coset algebra given by

$$
W=\frac{W_{1+\infty} \otimes \bar{W}_{1+\infty}}{\hat{U}(1)_{\text {diagonal }}}
$$

while for gap of topological origin we will have:

$$
W=W_{1+\infty} \otimes \bar{W}_{1+\infty} .
$$

It should be noticed that, for $c \geq 2$ for both (42) and (43), there are [19] ground states with the residual dynamical symmetry

$$
W=W_{m} \otimes \bar{W}_{m},
$$

where $W_{m}$ is the Fateev, Lykyanov, Zamolodchikov algebra [31] in the limit $C_{W_{m}} \rightarrow m-1$. These ground states describe unconventional superconductors whose excitation spectrum consists entirely of neutral, spinon excitations with non-Abelian fractional statistics and $S U(m)$ isospin symmetry. It should be observed that the $S U(m)$ symmetry of these excitations is different from the usual symmetry of, say, the quark model of strong interactions [32] since spinons do not come in the full $S U(m)$ multiplets but, rather, only the highest-weight state is present. However, they combine according to the usual $S U(m)$ fusion rules, which explains the non-Abelian character of their monodromies.

The examples discussed above clarify the fact that charge-spin separation and non-Abelian statistics are universal properties of $2 \mathrm{D}$ superconductors emerging only from incompressibility; the way in which incompressibility ( the gap) emerges- from topological order or SSBaffects, in fact, only the algebra of the edge excitations of the 2D incompressible fluid. It should by pointed out, however, that topological superconductors, described by 
Abelian mixed CS term, correspond to the generic representation of the $W_{1+\infty}$ algebra. It has been pointed out in [33] that, when an extra $Z_{2}$ symmetry is added, namely when the mixed CS term has a $U(1) \times U(1) \times Z_{2}$ symmetry, the excitations have non-Abelian statistics. Possible applications to topological superconductors are under investigation.

\section{SUMMARY AND CONCLUDING REMARKS}

In this paper we showed that, for superconducting systems with non trivial topologies, incompressibility alone suffices to guarantee both a ground state degeneracy and fractional statistics.

More important, we showed that the ground state degeneracy encodes relevant information about the microscopic mechanism originating the superconducting gap. In fact, when the gap emerges from SSB, the degeneracy is determined by the symmetry breaking $U(1) \rightarrow$ $Z_{N}$ and, thus, the Aharonov-Bohm interaction between charges and vortices is described by an effective mixed CS model with degeneracy $\left(k_{1} \times k_{2}\right)^{2}$ if $k=k_{1} / k_{2}$ is the CS coefficient. At variance, in the superconduting phase of a topological superconductor described by the $\mathrm{BF}$ model, there is only an electric condensate and the resulting ground state degeneracy is given by $k_{1} k_{2}$. This difference in the ground state degeneracy implies that the entanglement entropy of topological superconductors is half the one of standard superconductors. A measure of entanglement entropy may thus distinguish between the two different mechanisms giving rise to superconductivity.

Finally, since the BF model introduced in [5] describes also superinsulators in terms of a parity and time reversal invariant topological model, one may wonder if the analysis carried in this paper may be relevant to characterize relevant features of topological insulators [34] also; a topological insulator being a material which, while being an insulator in the bulk, allows for the motion of charges at its boundary yielding edge states which are topologically protected due to time-reversal invariance.
[1] For a review see: "The Quantum Hall Effect", R. E. Prange and S. M. Girvin eds., Springer Verlag, New York (1990).

[2] X.-G. Wen and Q. Niu, Phys. Rev. B 41 (1990) 9377; X.G. Wen Advances in Physics 44, 405 (1995), cond-mat/9506066, X.-G. Wen, Int. Jour. Mod. Phys. B 6 (1992) 1711, "Topological Orders and Edge Excitations in FQH states", MIT preprint 95-148; A. Zee, "From Semionics to Topological Fluids", in "Cosmology and Elementary Particles", Proceedings of the 1991 Rio Piedras Winter School, D. Altschuler, J. F. Nieves, J. P. de Leon and M. Ubriaco eds., World Scientific, Singapore (1991).

[3] For a review see: F. Wilczek, Fractional Statistics and Anyon Superconductivity, World Scientific, Singapore (1990).

[4] R. Jackiw and S. Templeton, Phys. Rev. D23 (1981) 2291; J. Schonfeld, Nucl. Phys. B185 (1981) 157; S. Deser, R. Jackiw and S. Templeton, Phys. Rev. Lett. 48 (1982) 975; Ann. Phys. (N.Y.) 140 (1982) 372.

[5] M. C. Diamantini, P. Sodano and C. A. Trugenberger, Eur. Phys.J. B 53, 19 (2006), DOI:10.1140/epjb/e200600345-0, hep-th/0511192 J. Phys. A39 (2006) 253.

[6] For a review see: D. Birmingham, M. Blau, M. Rakowski and G. Thompson, Phsy. Rep. 209 (1991) 129.

[7] M. C. Diamantini, P. Sodano and C. A. Trugenberger, Nucl. Phys. B448 (1995) 505; Nucl. Phys.B 4741 (1996) 64.

[8] M. Freedman, C. Nayak, K. Shtengel, K. Walker and Z. Wang, Ann. Phys. 310 (2004) 428.

[9] A. Stern, Phys. Rev.B 50 (1994) 10092; L.B. Ioffe, M.V. Feigel'man, A.S. Ioselevich, D. Ivanov, M. Troyer and G. Blatter, Nature 415 (2002) 503.

[10] Cirillo, private communication.

[11] A. Cappelli, C.A. Trugenberger and G. Zemba, Nucl. Phys.B 396,465 (1993);Phys. Rev. Lett 72 (1994) 1902;
Nucl. Phys.B 448 (1995) 470; Int. J. Mod. Phys. A12 (1997) 1101.

[12] A. Cappelli, L.S. Georgiev and I.T. Todorov, Nucl. Phys.B 599 (2001) 499.

[13] F.A. Bais, A. Morozov and M. de Wild Propitius, Phys. Rev. Lett 71 (1993) 2383.

[14] S.P. Kou, M. Levin and X.G. Wen, arXiv:0803.2300 M. Barkeshli and X.G. Wen, arXiv:0909.4882

[15] M. C. Diamantini, P. Sodano and C. A. Trugenberger, arXiv:1006.0412

[16] A. Vetergren, J. Lidmar and T.H. Hansson, Europhys. Lett. 69 (2005) 256.

[17] A. Kitaev and J. Preskill, Phys. Rev. Lett. 96 (2006) 110404; A. Levin and X.G. Wen, Phys. Rev. Lett. 96 110405.

[18] I. klich, G. Refael and A. Silva, Phys. Rev. A74 (2006) 032306; K. Le Hur, S. Rachel and F. Song, APS March Meeting 2010.

[19] C.A. Trugenberger, Nucl. Phys. B716 (2005) 509.

[20] D.B. Fairlie, P. Fletcher and C.K. Zachos, Phys. Rev B 218 (1989) 203; J. Math. Phys. 31 (1990) 1088. D.B. Fairlie and C.K. Zachos, Phys. Lett. B 224 (1989) 201.

[21] Dunne and C.A. Trugenberger Mod. Phys. Lett. A4 (1989) 1635; A.P. Polychronakos, Phys. Lett. B 241 (1990) 37; X.G. Wen, Phys. Rev. B40 (1989) 7387.

[22] I.I. Kogan, ,Int. J. Mod. Phys. A 9 (1994) 3887.

[23] M. Bergeron, G.W. Semenoff and R.J. Szabo, Nucl. Phys. B437 (1995) 695.

[24] P. Fendley, M.P.A. Fisher and C. Nayak, J. Stat. Phys. 126 (2007) 1111.

[25] E.P. Verlinde Nucl. Phys. B300 (1988) 360.

[26] S. Gukov, E. Martinec, G. Moore and A. Strominger, hep-th/0403225

[27] V. Kac and A. Radul, Commun. Math. Phys. 157 (1993) 429. 
[28] E. Witten, Comm. Math. Phys. 121 (1989) 351.

[29] A.P. Balachandran, G. Bimonte, K.S. Gupta and A. Stern, Int. J. Mod. Phys. A7 ((1992) 4655 ; M. Banados, Phys. Rev. D10 (1995) 5816.

[30] R. Floreanini and R. Jackiw, Phys. Rev. Lett. 59 (1987) 1873.

[31] V.A. Fateev and A.B. Zamolodchikov, Nucl. Phys. B280 (1987) 644; V.A. Fateev and S. L. Lykyanov, Int. J. Mod.
Phys. A3 (1988) 507.

[32] See e.g. : H. i, Lie Algebras in Particle Physics, AddisonWesley, Reading (1982).

[33] M. Barkeshli and X.G. Wen, arXiv:0909.4882.

[34] L. Fu, C.L. Kane and E.J. Mele, Phys. Rev. Lett. 98 (2007) 106803. 